\title{
Deformation of giant lipid bilayer vesicles in shear flow
}

\author{
K. H. de Haas, C. Blom, D. van den Ende, M. H. G. Duits, and J. Mellema \\ Rheology Group, Department of Applied Physics, J. M. Burgers Centre, University of Twente, P.O. Box 217, 7500 AE Enschede, \\ The Netherlands \\ (Received 14 March 1997)
}

\begin{abstract}
We describe experimental studies of the deformation of giant lipid bilayer vesicles in shear flow. The experiments are carried out with a counterrotating Couette apparatus. The deformation depends on the mechanical properties of the lipid bilayer, the vesicle radius, and the viscosity of the surrounding Newtonian liquid. We show that the relevant mechanical parameter is the bending rigidity. A simple model has been developed that describes the deformation of a vesicle. This model takes thermal undulations of the bilayer into account. We have obtained a value for the bending rigidity of dimyristoyl-phosphatidylcholine bilayers and its value has been compared with literature data and with results from micropipette aspiration experiments. From the measurements we are able to discriminate between unilamellar and multilamellar vesicles.
\end{abstract} [S1063-651X(97)12212-7]

PACS number(s): 87.22.Bt, 68.10.Cr, 82.65.Dp, 83.50.-v

\section{INTRODUCTION}

Early experiments on the deformation of liquid droplets immersed in another liquid were carried out by Taylor [1] with a parallel-band apparatus. He also developed a theory for small deformations of droplets [2] where the interface is characterized through the surface tension. Subsequently, the deformation of red blood cells in a shear flow was investigated; see, e.g., [3]. These experiments were carried out with a transparent cone-plate rheometer and recently also with a counterrotating Couette apparatus [4]. Also recently, the deformation of synthetic polymeric capsules has been measured [5]. In all cases the mechanical interfacial properties of interest were the surface shear modulus and a surface viscosity.

Vesicles are liquid droplets immersed in a liquid, with an interface that consists of a lipid bilayer. Lipid bilayers are in the ordered gel state when temperature is below the critical or phase-transition temperature $T_{c}$; see, e.g., [6]. Above $T_{c}$ the bilayer is in the liquid-crystalline state. The lipids are disordered and can move freely through the bilayer.

We report deformation experiments on lipid bilayer vesicles in shear flow. These experiments have been carried out with a counterrotating Couette apparatus that is presented in [7]. In this study the lipid dimyristoyl-phosphatidylcholine (DMPC) has been selected $\left(T_{c}=23^{\circ} \mathrm{C}\right)$. In these experiments temperature is fixed at $30^{\circ} \mathrm{C}$, so the bilayer is in the fluid state. With our apparatus no significant deformation can be observed when the bilayer is in the gel state.

For a fluid lipid bilayer, a surface shear modulus is not expected to exist, except at very short time scales [8]. In a steady shear flow the time scales are relatively long. The elastic behavior is generally governed by a dilatation modulus and the presence of thermal undulations. In the description of these undulations, the relevant mechanical parameter is the curvature modulus or bending rigidity. We assume that dilatation of the lipid bilayer can be neglected. This assumption will be discussed with the experimental results. Thus the vesicles are assumed to have a constant area. Also, the volume of the vesicle can be assumed to be constant since the bilayer is impermeable to water on the experimental time scale. The vesicle area is larger than that of a sphere with the same volume by an amount that will be called the excess area. In the physical description of the membrane, an effective surface tension comes into play, which depends on the total excess area.

We have combined a linear theory for the deformation of a liquid droplet with an elastic interface in shear flow with a theory for thermal membrane undulations in order to obtain a simple deformation model. These models and the derivation of the present model are outlined in Sec. II, followed by a short description of the synthesis of the vesicles in Sec. III. Next, in Sec. IV, we will present the experimental results. The deformation behavior of spherical and nonspherical vesicles will be presented. Our model will be applied to the case where vesicles are initially spherical. From this we are able to obtain a value for the bending rigidity. Furthermore, the distinction between unilamellar and multilamellar bilayers can be made. We also obtain values for the effective surface tension of the lipid bilayer. We conclude in Sec. V.

\section{THEORY}

\section{A. Thermal undulations of a vesicle bilayer}

In this section we present a brief review of the description of the dynamics of a vesicle surface. It is based on the articles of Milner and Safran [9] and van der Linden, Bedeaux, and Borkovec [10]. The vesicle shape is represented by an expansion in spherical harmonics [9]:

$$
r(\theta, \phi, t)=a\left(1+\sum_{l, m} u_{l m}(t) Y_{l m}(\theta, \phi)\right),
$$

with $Y_{l m}(\theta, \phi)$ the normalized spherical harmonic functions, $u_{l m}(t)$ their amplitudes, $l=0,1,2, \ldots$, and $-l \leqslant m \leqslant l$. The apparent radius $a$ is determined under the assumption that the vesicle volume is constant: 


$$
V=\frac{4 \pi}{3} a^{3}
$$

The vesicle area is given by

$$
A=4 \pi a^{2}+\frac{a^{2}}{2} \sum_{l=2}\left|u_{l m}\right|^{2}(l+2)(l-1) .
$$

Third- and higher-order terms in $u_{l m}$ are neglected. As mentioned previously, we assume that the total area $A$ is constant.

The potential energy of a bilayer with surface tension $\sigma$ and bending rigidity $k_{c}$ is given by Helfrich's expression [11]

$$
U=\int\left[\sigma+\frac{1}{2} k_{c}\left(C-C_{0}\right)^{2}\right] d A,
$$

with $C$ the curvature of the surface and $C_{0}$ the natural curvature of the surface. The surface tension is a Lagrange multiplier to ensure that the surface area remains constant.

From the Boltzmann equipartition theorem we are now able to determine the amplitude of each spherical harmonic mode because the vesicle is placed in a thermal reservoir. The energy that is stored in each mode equals $\frac{1}{2} k T$, with the Boltzmann constant $k$ and temperature $T$. We restrict this description to small undulation amplitudes and obtain [9]

$$
\begin{aligned}
\left\langle\left|u_{l m}\right|^{2}\right\rangle= & k T\left(( l + 2 ) ( l - 1 ) \left\{k_{c}\left[l(l+1)-4 w+2 w^{2}\right]\right.\right. \\
& \left.\left.+\sigma a^{2}\right\}\right)^{-1}
\end{aligned}
$$

with $w=\frac{1}{2} a C_{0}$. The mean excess area is then

$$
\frac{A_{\mathrm{exc}}}{A}=\frac{A-4 \pi a^{2}}{A}=\frac{k T}{8 \pi} \sum_{l=2}^{l_{\max }} \frac{2 l+1}{k_{c}\left[l(l+1)-4 w+2 w^{2}\right]+\sigma a^{2}} .
$$

The upper cutoff is $l_{\max } \equiv \pi a / b$, with $b$ a microscopic length. Its smallest value is on the order of molecular dimensions because it is a continuum description. For large $l_{\max }$ we can rewrite Eq. (6) as an integral:

$$
\begin{aligned}
\frac{A_{\mathrm{exc}}}{A} & =\frac{k T}{8 \pi} \int_{2}^{l_{\max }} \frac{2 l+1}{k_{c}\left[l(l+1)-4 w+2 w^{2}\right]+\sigma a^{2}} d l \\
& =\frac{k T}{8 \pi k_{c}} \ln \left(\frac{\frac{\pi^{2}}{b^{2}}+\frac{\sigma}{k_{c}}}{\frac{4 \pi\left(6-4 w+2 w^{2}\right)}{A}+\frac{\sigma}{k_{c}}}\right),
\end{aligned}
$$

where we used $l_{\text {max }}^{2} \gg l_{\text {max }}-4 w+2 w^{2}$ because we expect that $w$ is of the order of unity or less. The right-hand side resembles the Helfrich equation for thermal undulations in a flat square lipid membrane [12]:

$$
\frac{A_{\mathrm{exc}}}{A}=\frac{k T}{8 \pi k_{c}} \ln \left(\frac{\frac{\pi^{2}}{b^{2}}+\frac{\sigma}{k_{c}}}{\frac{\pi^{2}}{A}+\frac{\sigma}{k_{c}}}\right),
$$

with $b$ the same microscopic length as in the quasispherical description. Usually we have

$$
\frac{\pi^{2}}{b^{2}} \gg \frac{\sigma}{k_{c}} \gg \frac{\pi^{2}}{A},
$$

with $b \approx 10^{-9} \mathrm{~m}[12], \quad \sigma \approx 10^{-8}-10^{-3} \mathrm{Nm}^{-1}$ [13], $k_{c}$ $\approx 10^{-19} \mathrm{~J}$ [13], and $A \approx 10^{-9} \mathrm{~m}^{2}$. When Eq. (9) is valid, representations (7) and (8) are equivalent.

\section{B. Excess area of a deformed vesicle}

In Sec. II A we obtained an expression for the excess area of a lipid bilayer vesicle at rest. It is based on thermal equilibrium and equipartition of energy for each undulation mode. When a vesicle is deformed in a shear flow we have a different situation because it is not at equilibrium anymore. The lipid bilayer rotates around the vesicle interior and is kept at a constant temperature. We assume that equipartition may be used for the deformed lipid bilayer. Under a microscope we observe the apparent vesicle shape. We assume that this shape is a projection of an ellipsoid or the projection of a linear combination of second-order spherical harmonic functions. The vesicle area $A$ is supposed to be constant and therefore the area is given by

$$
A=A_{e}^{\mathrm{exc}}+A_{e}
$$

whereas for the spherical vesicle at rest

$$
A=A_{s}^{\mathrm{exc}}+A_{s},
$$

with $A_{s}$ and $A_{e}$ the areas of a sphere and an ellipsoid, respectively. Setting both equations equal to each other and using Eq. (6), we obtain

$$
\begin{aligned}
\frac{A_{e}-A_{s}}{A}= & \frac{k T}{8 \pi} \sum_{l=2}^{l_{\max }} \frac{2 l+1}{k_{c}\left[l(l+1)-4 w+2 w^{2}\right]+\sigma_{e} a^{2}} \\
& -\frac{k T}{8 \pi} \sum_{l^{\prime}=2}^{l_{\max }} \frac{2 l^{\prime}+1}{k_{c}\left[l^{\prime}\left(l^{\prime}+1\right)-4 w+2 w^{2}\right]+\sigma_{s} a^{2}} .
\end{aligned}
$$

The surface tension is considered to be a Lagrange multiplier, and from this equation it follows that $\sigma_{e}$ is larger for the deformed vesicle than for a spherical vesicle $\sigma_{s}$ at rest. A new equilibrium is found after deformation and the amplitudes of the different modes settle at different values.

For large $l_{\max }$ we can rewrite Eq. (12) as an integral, which results in

$$
\begin{aligned}
\frac{A_{e}-A_{s}}{A}= & \frac{k T}{8 \pi k_{c}} \ln \left[\left(\frac{4\left(6-4 w+2 w^{2}\right) \pi k_{c}+A \sigma_{s}}{4\left(6-4 w+2 w^{2}\right) \pi k_{c}+A \sigma_{e}}\right)\right. \\
& \left.\times\left(\frac{\pi^{2} k_{c}+b^{2} \sigma_{e}}{\pi^{2} k_{c}+b^{2} \sigma_{s}}\right)\right] .
\end{aligned}
$$

With the estimates (9) we finally obtain

$$
\frac{A_{e}-A_{s}}{A}=\frac{k T}{8 \pi k_{c}} \ln \left(\frac{\sigma_{e}}{\sigma_{s}}\right) \text {. }
$$




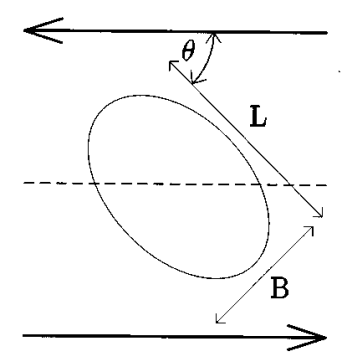

FIG. 1. Image of a deformed vesicle. The dashed line marks the plane of zero velocity.

With Eq. (9) we obtain exactly the same expression as from Helfrich's model for flat membranes.

\section{Vesicle deformation model in shear flow}

The deformation of an initially spherical vesicle is described by the Taylor deformation parameter $D$,

$$
D=\frac{L-B}{L+B},
$$

where $L$ and $B$ are the lengths of the major and minor axes of the deformed vesicle, respectively. Figure 1 shows a schematic image of the projection of a deformed vesicle. The average vesicle shape in a shear flow can be written as

$$
\langle r(\theta, \phi)\rangle=r^{\prime}+2 r^{\prime} D \sin ^{2} \theta \sin \phi \cos \phi,
$$

where the effective radius $r^{\prime}$ depends slightly on $D$. This is the theoretical shape of an elastic capsule (or liquid droplet) for small deformations in shear flow [14]. According to Eq. (1), this shape can be represented by a linear combination of spherical harmonic functions

$$
\begin{aligned}
r(\theta, \phi, t)= & a\left[1+u_{00} Y_{00}(\theta, \phi)+u_{2,-2} Y_{2,-2}(\theta, \phi)\right. \\
& \left.+u_{22} Y_{22}(\theta, \phi)\right],
\end{aligned}
$$

with $u_{00}, u_{2,-2}$, and $u_{22}$ functions of the deformation parameter. With the conserved vesicle volume assumption for this shape, the apparent vesicle area is related to $D$ through

$$
\frac{A_{e}-A_{s}}{A_{s}}=\frac{8 D^{2}}{15}
$$

which follows directly from Eq. (3). To first order in the relative excess area, the quantity $\left(A_{e}-A_{s}\right) / A_{s}$ equals $\left(A_{e}\right.$ $\left.-A_{s}\right) / A$. The substitution of Eq. (18) into Eq. (14) gives the coupling between our experiment and the undulation theory.

Next the relation between the deformation parameter and shear rate remains to be evaluated. We have derived an expression based on the work of Oldroyd $[15,16]$. His model describes the viscoelastic behavior of a monodisperse dispersion of elastic capsules with negligible wall thicknesses that are immersed in a Newtonian liquid. It is a cell model based on Stokes's equations and is valid for small deformations. The shape of a single deformed vesicle in a steady flow is recovered by taking the limits of zero vesicle volume fraction and zero frequency. A straightforward calculation gives

$$
D=a \eta \dot{\gamma} \frac{5 \mu+15 \kappa}{8 \mu \sigma+8 \mu \kappa+12 \sigma \kappa},
$$

with $\dot{\gamma}$ the shear rate, $\sigma$ the surface tension, $\kappa$ the dilatation modulus, $\mu$ the shear modulus, and $\eta$ the viscosity of the exterior liquid. The shape of the vesicle is described by Eq. (17). The shear modulus has to be set equal to zero and we find

$$
D=\frac{5 a \eta}{4 \sigma} \dot{\gamma} .
$$

Oldroyd does not consider the influence of the bending rigidity, but for $\sigma \gg k_{c} / a^{2}$ its contribution is negligible [14]. For the previously given estimates this is indeed the case.

We will use Eq. (20) for the description of the deformation of a vesicle as a function of shear rate. Previously it was argued that the surface tension $\sigma_{e}$ of the lipid bilayer varies with the amount of excess area through Eq. (14). This surface tension is substituted into Eq. (20) with $\sigma=\sigma_{e}$. The apparent vesicle area is eliminated by substitution of Eq. (18) into Eq. (14) and the following relation between the deformation parameter and shear rate results:

$$
\dot{\gamma}=\frac{4 \sigma_{s} D}{5 a \eta} \exp \left(\frac{64 \pi k_{c}}{15 k T} D^{2}\right) .
$$

Fits of Eq. (21) to the experimental data give the $\sigma_{s}$ and $k_{c}$ values.

\section{EXPERIMENT}

Dimyristoyl-phosphatidylcholine lipids are purchased from Lipid Products Ltd. United Kingdom and are used without further purification. The vesicles are prepared with a hydration method similar to that of Reeves and Dowben [17]. A lipid solution in a mixture of chloroform and methanol is dried by evaporation. The remaining film is gently brought in contact with distilled water, a low-ionic salt solution, or another nonionic solution. The lipids swell by the intake of water and with time giant vesicles are formed spontaneously.

The main advantage of this method is that vesicles with diameters of more than $50 \mu \mathrm{m}$ can be formed. Furthermore, the method is very simple. The main drawback is that multilamellar vesicles and, depending on the conditions, nonvesicular structures are formed in addition to unilamellar vesicles.

We have stimulated the formation of unilamellar vesicles in a way similar to the synthesis described in [18]. The surface of a thin Teflon disk with a $1.6 \mathrm{~mm}$ diameter is roughened with emery paper. It is put in a glass bottle and kept at $37^{\circ} \mathrm{C}$. About $0.05 \mathrm{ml}$ of a $10-\mathrm{mg} / \mathrm{ml}$ DMPC solution is spread out across its surface and the chloroform-methanol mixture evaporates almost immediately. The film is next dried for a few hours. Then the disk is covered gently with a few centimeters of distilled water at $37^{\circ} \mathrm{C}$. The sample is stored for $24 \mathrm{~h}$ at $37^{\circ} \mathrm{C}$. After a few hours a white cloud can be observed. This covers the entire Teflon disk. It grows to a thickness of about $1 \mathrm{~mm}$ over $24 \mathrm{~h}$. The vesicle cloud is dispersed in water by gently shaking the sample. This dispersion is further diluted before use. 


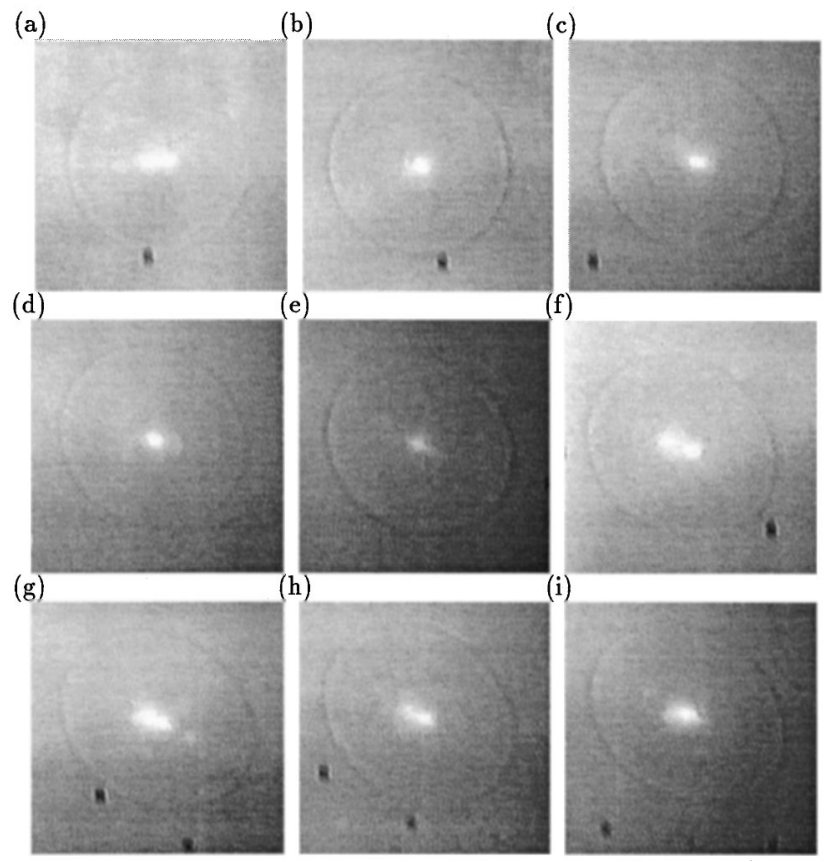

FIG. 2. Examples of a deformed vesicle with a $55 \mu \mathrm{m}$ radius: (a) $\dot{\gamma}=0.0 \mathrm{~s}^{-1}, \quad$ (b) $\dot{\gamma}=1.0 \mathrm{~s}^{-1}$, (c) $\dot{\gamma}=2.0 \mathrm{~s}^{-1}$, (d) $\dot{\gamma}$ $=4.0 \mathrm{~s}^{-1}$, (e) $\dot{\gamma}=6.0 \mathrm{~s}^{-1}$, (f) $\dot{\gamma}=8.0 \mathrm{~s}^{-1}$, (g) $\dot{\gamma}=10.0 \mathrm{~s}^{-1}$, (h) $\dot{\gamma}=12.0 \mathrm{~s}^{-1}$, and (i) $\dot{\gamma}=15.0 \mathrm{~s}^{-1}$.

\section{RESULTS}

\section{A. Observation of deformed vesicles}

The vesicles can be distinguished into two groups: (i) vesicles that are initially spherical and (ii) vesicles that are initially nonspherical.

(i) At low shear rates, the deformation of an initially spherical vesicle is too small to observe anything but a rotation of the vesicle. At sufficiently high shear rates it deforms into a steady shape with a fixed orientation. For small deformations this orientation is about $45^{\circ}$ with respect to the streamlines. The orientation decreases with increasing shear rate. We observe tank treading: The membrane circulates around the interior of the vesicle. An example of deformation of an initially spherical vesicle is given in Fig. 2. With a radius of about $55 \mu \mathrm{m}$ it is one of the largest vesicles used. The vertical direction in the pictures is the radial direction in the gap. The inner cylinder is situated at the upper side and the outer cylinder at the lower side of each picture. The fluid at the upper side moves to the left and at the lower side it moves to the right. These images have been digitized with a frame grabber and the quality of the bit maps has been improved.

(ii) At low shear rates, an initially nonspherical vesicle deforms slightly and undergoes a periodic flipping motion comparable to the motion of rigid ellipsoids. The vesicle rotates around its center, but the angular velocity depends on its orientation with respect to the streamlines. When the shear rate is increased, the vesicle starts to oscillate while it is still rotating. The deformation parameter is now a periodic function in time and is largest when the orientation is about $45^{\circ}$ and smallest when it is $-45^{\circ}$. As is the case with initially spherical vesicles at sufficiently high shear rates, the vesicle changes into a steady deformed shape with a fixed

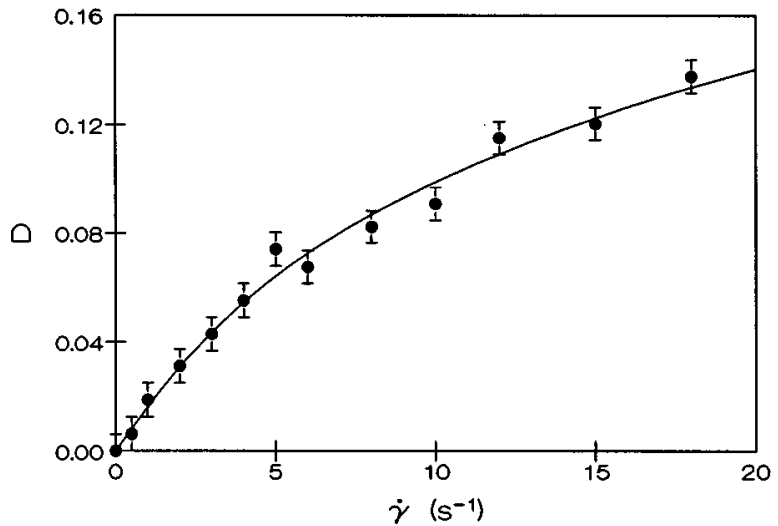

FIG. 3. Deformation parameter of the vesicle from the previous example.

orientation and tank treading is observed.

Our observations agree with observations reported in literature for emulsion droplets, red blood cells, and synthetic capsules. The deformation of an initially spherical vesicle resembles the deformation of an emulsion drop. The flipping motion of nonspherical vesicles also has been observed when a sufficiently small shear stress is applied to red blood cells (see, e.g., [19]), for which a similar transition to a steady deformed shape with a fixed orientation, and tank treading, are also observed. The oscillating behavior has also been observed with synthetic capsules [5].

\section{B. Application of the deformation model}

A typical result for the shear rate dependence on the deformation parameter of an initially spherical vesicle is given in Fig. 3. This is the same vesicle that is shown in Fig. 2. The possible uncertainty is determined by the size of the vesicle with respect to the size of the window of observation. The deformation parameter is a nonlinear function of shear rate. The least-squares fit to Eq. (21) is shown. We have measured the deformation of 26 vesicles that were initially spherical. For each vesicle we have obtained the bending rigidity and the effective surface tension of the undeformed vesicle. The bending rigidities are displayed in Fig. 4. For convenience, we plot the bending rigidity versus the vesicle radius. We see that the experimental error can be rather large.

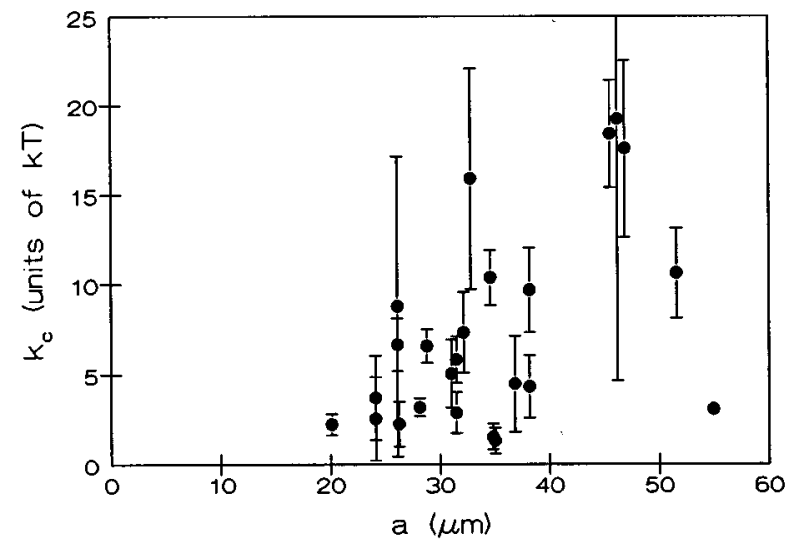

FIG. 4. Bending rigidity of 26 vesicles. 


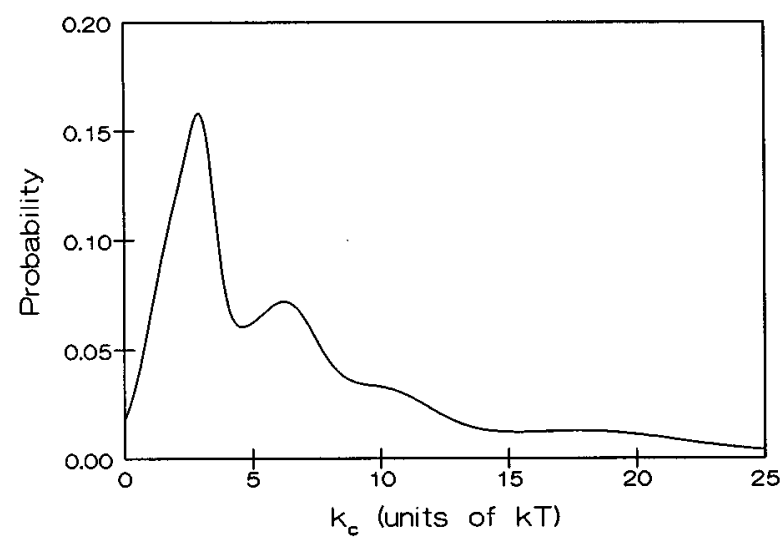

FIG. 5. Distribution of the bending rigidity.

Representing each value of $k_{c}$ by a normalized Gaussian probability distribution function with a width determined by the uncertainty in $k_{c}$, we obtain the distribution of Fig. 5 when we add up all distributions. This distribution suggests the presence of a discretization in the possible values of the bending rigidity, where the first peak may be due to unilamellar vesicles, the second due to bilamellar vesicles, and so on. Thus, for unilamellar DMPC bilayers, we obtain $k_{c}$ $=3.0 \pm 0.3 \mathrm{kT}=(1.3 \pm 0.1) \times 10^{-20} \mathrm{~J}$. This is somewhat lower than the literature values for $k_{c}:(3.5-6.5) \times 10^{-20} \mathrm{~J}$ at $26^{\circ} \mathrm{C}[20], 5.6 \times 10^{-20} \mathrm{~J}$ at $29^{\circ} \mathrm{C}[21], 11.5 \times 10^{-20} \mathrm{~J}$ at $30{ }^{\circ} \mathrm{C}$ [22], and $24 \times 10^{-20} \mathrm{~J}$ at $26^{\circ} \mathrm{C}$ [23]. The second literature value was determined from a micropipette experiment and the other values were obtained with vesiclecontour spectroscopy. For both methods also a distinction between unilamellar and multilamellar vesicles was also made. In the vesicle-contour spectroscopy experiments a distribution like that in Fig. 5 was found. These experiments result in sharper peaks due to a smaller experimental error. Duwe and Sackmann [22] observe that small amounts of a short bipolar lipid may reduce the bending rigidity down to the order of one kT. The only possibility that might explain our relatively low $k_{c}$ value is the possible presence of trace impurities in our lipids. We have tested the lipid batch with thin-layer chromatography and observed a weak second spot, indicating the presence of a small fraction of impurities. In addition, we have carried out a micropipette aspiration experiment, which resulted in comparable low values for $k_{c}$ while the dilatation modulus $\kappa$ also turned out to be somewhat lower than the literature values [21]. This experiment is described in Ref. [24].

We calculated the effective surface tension $\sigma_{s}$ and the results are presented in Fig. 6. The values range from $10^{-7}$ to $10^{-5} \mathrm{Nm}^{-1}$. Kummrow and Helfrich [25] measured the deformation of lipid vesicles under the influence of an electric field and determined the effective tension for separate vesicles. These values are on the order of $10^{-9} \mathrm{Nm}^{-1}$. They mention that vesicles look rigid above $\sigma_{s}=10^{-6} \mathrm{Nm}^{-1}$, i.e., the thermal undulations are not visible to the eye, as was the case for our vesicles.

It is possible to determine the effective tension $\sigma$ during deformation with Eq. (14). At most, it is one order of magnitude larger than $\sigma_{s}$. Therefore, the assumption of negli-

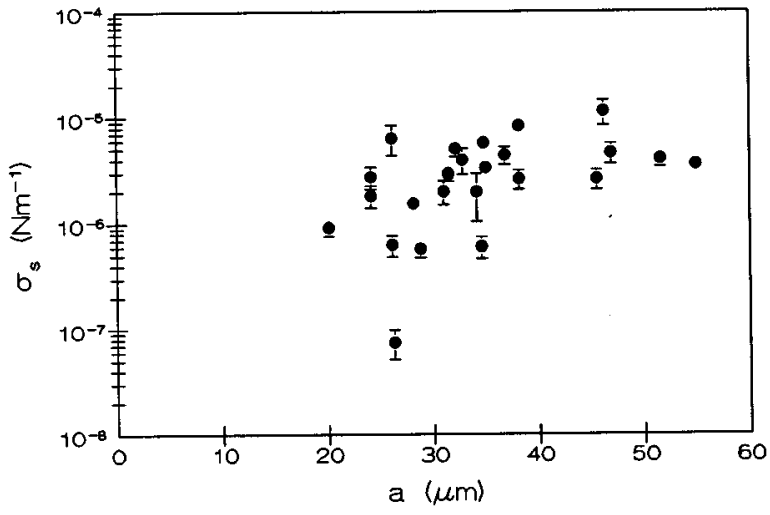

FIG. 6. Effective surface tension of the undeformed vesicle.

gible dilatation is correct. The increase of the real area due to dilatation is given by

$$
\frac{\Delta A_{d}}{A}=\frac{\sigma_{e}-\sigma_{s}}{\kappa} .
$$

Literature values of the dilatation modulus are on the order of $0.1 \mathrm{Nm}^{-1}$. We have verified those values with a micropipette experiment; see [24]. We found values of about $0.05 \mathrm{Nm}^{-1}$. A comparison of Eqs. (22) and (14) with estimates of $\sigma_{e}, \sigma_{s}$, and $\kappa$ shows that the increase of area induced by dilatation is at least one order of magnitude smaller than the apparent area increase. Furthermore, we conclude that our assumption (9) holds for our $k_{c}$ and $\sigma_{s}$ values. Thus application of Eq. (14) is allowed in our case.

\section{CONCLUSIONS}

We have measured the deformation of giant DMPC bilayer vesicles in shear flow. The measurements have been carried out with a counterrotating Couette apparatus that we developed recently. The qualitative deformation behavior of initially spherical vesicles corresponds to that of emulsion droplets. The qualitative deformation behavior of initially nonspherical vesicles corresponds to that of red blood cells and synthetic polymeric capsules.

We have developed a simple model that describes the deformation parameter as a function of shear rate. It is a combination of a linear theory of liquid droplets with an elastic interface and a theory that describes the vesicle interface in terms of thermal undulations. From this model it becomes clear that at low shear rates the deformation behavior is mainly due to the dynamics of the undulating surface, while dilatation of the surface is negligible.

We have estimated the bending rigidity to be $k_{c}=(1.3$ $\pm 0.1) \times 10^{-20} \mathrm{~J}$. We learned from the distribution of the bending rigidities for the separate vesicles that many of them are not unilamellar. Multilamellar vesicles may be expected because of the nature of the preparation method. The observation of a discretization in the bending rigidities for each vesicle has also been observed in various experiments that were reported in literature. In future experiments, purified lipids need to be used for determining the influence of impurities in the bilayer on the bending rigidity. 
[1] G. I. Taylor, Proc. R. Soc. London, Ser. A 146, 501 (1934).

[2] G. I. Taylor, Proc. R. Soc. London, Ser. A 138, 41 (1932).

[3] T. Fischer and H. Schmidt-Schönbein, Blood Cells 3, 351 (1977)

[4] G. J. Streekstra, Ph.D. thesis, Utrecht University, 1994 (unpublished).

[5] K. S. Chang and W. L. Olbricht, J. Fluid Mech. 250, 609 (1993).

[6] G. Cevc and D. Marsh, Phospholipid Bilayers (Wiley, New York, 1987).

[7] K. H. de Haas, D. van den Ende, C. Blom, E. G. Altena, G. J. Beukema, and J. Mellema (unpublished).

[8] J. B. A. F. Smeulders, C. Blom, and J. Mellema, Phys. Rev. A 42, 3483 (1990).

[9] S. T. Milner and S. A. Safran, Phys. Rev. A 36, 4371 (1987).

[10] E. van der Linden, D. Bedeaux, and M. Borkovec, Physica A 162, 99 (1989).

[11] W. Helfrich, Z. Naturforsch. C 28, 693 (1973).
[12] W. Helfrich and R. M. Servuss, Nuovo Cimento D 3, 137 (1984).

[13] E. Evans and D. Needham, J. Phys. Chem. 91, 4219 (1987).

[14] Y. Fujitani, Fluid Dyn. Res. 15, 1 (1995).

[15] J. G. Oldroyd, Proc. R. Soc. London, Ser. A 218, 122 (1953).

[16] J. G. Oldroyd, Proc. R. Soc. London, Ser. A 232, 567 (1955).

[17] J. P. Reeves and R. M. Dowben, J. Cell Physiol. 73, 49 (1969).

[18] D. Needham and E. Evans, Biochemistry 27, 8261 (1988).

[19] S. R. Keller and R. Skalak, J. Fluid Mech. 120, 27 (1982).

[20] H. Engelhardt, H. P. Duwe, and E. Sackmann, J. Phys. (France) Lett. 46, 395 (1985).

[21] E. Evans and W. Rawics, Phys. Rev. Lett. 64, 2094 (1990).

[22] H. P. Duwe and E. Sackmann, Physica A 163, 410 (1990).

[23] G. Beblik, R. M. Servuss, and W. Helfrich, J. Phys. (France) 46, 1773 (1985).

[24] K. H. de Haas, Ph.D. thesis, University of Twente, 1997 (unpublished).

[25] M. Kummrow and W. Helfrich, Phys. Rev. A 44, 8356 (1991). 